\title{
The use of remdesivir outside of clinical trials during the COVID-19 pandemic
}

\author{
Vesa Halimi ${ }^{1}$, Armond Daci ${ }^{2}$, Nevenka Ridova ${ }^{3}$, Irina Panovska-Stavridis ${ }^{3}$, Milena Stevanovic ${ }^{4}$, Venko Filipce ${ }^{5}$, \\ Aleksandar Dimovski ${ }^{1}$ and Aleksandra Grozdanova ${ }^{1 *}$ (i)
}

\begin{abstract}
With a scientific background from filoviruses, paramyxoviruses, SARS-CoV, and MERS-CoV, remdesivir entered into the COVID-19 battle to become one of the favorable therapeutic candidates with potential antiviral activity in the treatment of this disease. Globally, remdesivir was accessed and investigated through clinical research (clinical trials) and clinical practice (compassionate use, expanded access, early access scheme, and emergency use). Currently, remdesivir approval status differs between states. This paper aims to review and analyze regulatory approaches for accessing and investigating remdesivir, by communicating regulatory variability between countries in terms of terminology, modalities, and protocols.
\end{abstract}

Keywords: COVID-19, Remdesivir, Clinical practice, Clinical trials, Expanded access, Compassionate use, Early access scheme, Emergency use, Conditional approval

\section{Introduction}

The undisputable medical need to find an effective therapeutic in the middle of COVID-19 pandemic, aligned regulatory authorities, clinicians, researchers, and manufacturers to consider using unapproved therapeutics or unapproved indications of already approved therapeutics with potential antiviral activity [1]. The main goal of finding an effective therapy is not only to determine the clinical efficacy and safety but also to determine the treatment duration, sensitive patients, and to ensure equitable patient access. As clinical trials began to show their preliminary outcomes, the established regulatory approaches to ensure the access to potential COVID-19 therapeutics have been reviewed, and as a consequence, some therapeutics have been revoked and are not recommended for use anymore $[2,3]$.

For instance, the rapid approval of emergency use of hydroxychloroquine and chloroquine in the USA on 28

\footnotetext{
* Correspondence: agrozdanova@ff.ukim.edu.mk

${ }^{1}$ Faculty of Pharmacy, University Ss. Cyril and Methodius University, Skopje, North Macedonia

Full list of author information is available at the end of the article
}

March 2020 [4], consequently revoked on 15 June 2020 [2], for safety and efficacy reasons, can be justified to some extent for the fact that these two antimalarial drugs had an already known safety profile in their approved indication(s). Also, the availability of scientific evidence in terms of safety and efficacy prompted FDA to decide that the potential benefits of hydroxychloroquine and chloroquine outweigh their potential risks, considering that no effective therapy was approved at that time [5]. On the other hand, several immunomodulating therapeutics like dexamethasone $[6,7]$, interleukin-1 inhibitors $[8,9]$, interleukin-6 inhibitors [10-12], interferon-beta [13], Bruton's tyrosine kinase inhibitors [14], Janus kinase inhibitors [15], have raised hope in the treatment of COVID-19. But with regard to remdesivir, some of the pertinent questions include how was remdesivir accessed in patients outside of clinical trials? How regulatory authorities and Gilead have communicated these regulatory approaches to clinicians? How the preliminary results of RCTs have affected the expansion of remdesivir's use in clinical practice, and later, its approval?

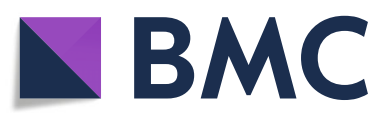

(c) The Author(s). 2020 Open Access This article is licensed under a Creative Commons Attribution 4.0 International License, which permits use, sharing, adaptation, distribution and reproduction in any medium or format, as long as you give appropriate credit to the original author(s) and the source, provide a link to the Creative Commons licence, and indicate if changes were made. The images or other third party material in this article are included in the article's Creative Commons licence, unless indicated otherwise in a credit line to the material. If material is not included in the article's Creative Commons licence and your intended use is not permitted by statutory regulation or exceeds the permitted use, you will need to obtain permission directly from the copyright holder. To view a copy of this licence, visit http://creativecommons.org/licenses/by/4.0/. The Creative Commons Public Domain Dedication waiver (http://creativecommons.org/publicdomain/zero/1.0/) applies to the data made available in this article, unless otherwise stated in a credit line to the data. 


\section{Aims and methods to evaluate data on remdesivir} The main objective of this paper is to elaborate and evaluate regulatory approaches for the use of remdesivir outside of clinical trials. We analyzed and relied on the scientific opinions and recommendations for remdesivir published by European Medicines Agency (EU-EMA) [16], Food and Drug Administration (US-FDA) [4] and Medicines and Healthcare products Regulatory Agency (UKMHRA) [17]. We compared and analyzed the data with the Gilead's protocol for compassionate use that is available on the website of the Italian Regulatory Agency (AIFA) [18]. The regulatory guidance and materials $[16,19-30]$ (Tables 1 and 2) were compared with peer-reviewed original articles considering compassionate use of remdesivir [31].
Two expanded access trials NCT04323761 [19] and NCT04302766 [20], as well as RCTs including NCT04257656 [32], NCT04292899 [33], NIAID trial [34], Inserm discovery trial, solidarity trial, and peerreviewed articles automatically indexed to these trials, were analyzed [32-34]. As a support, we used the Gilead's formal website to evaluate communication towards the public [35].

The access to remdesivir outside of clinical trials

Based on the in vitro and animal studies from SARS$\mathrm{CoV}$ and MERS-CoV as well as phase 1 clinical trials in healthy volunteers in EBOV, Gilead recommended the dosage and duration of treatment for remdesivir as 200 $\mathrm{mg}$ on day 1 followed by $100 \mathrm{mg}$ from day 2 to $10 \mathrm{de}-$ pending on the clinical assessment [36]. The access of

Table 1 Legal basis, terminologies, and regulatory evidence for compassionate use, expanded access, and for early access scheme in COVID-19

\begin{tabular}{ll}
\hline Regulatory agency & Legal basis \\
& \\
\hline Food and Drug Administration, & 21 CFR 312 via 21CFR 312.300, \\
FDA, USA & 21CFR 312.305, 21CFR 312.310, \\
& 21CFR 312.315, 21CFR312.320
\end{tabular}

Medicines and Healthcare Products Regulatory Agency (MHRA), UK
Human Medicines Regulations 2012 (SI 2012/1916)

\section{Terminology and other modalities}

Expanded access for individual patients, in emergency use, for intermediate-size population, for widespread treatment use.

Early access to medicines intended for more patients
European Medicines Agency

EMA, European Union
Article 83 of Regulation (EC) No. 726/2004
Compassionate use is intended for a group of patients, although the approval, terminology and modalities are left within the remit of the National European Agencies.
Additional regulatory evidence for the use remdesivir in COVID-19, outside of clinical trials

NCT04323761 treatment
IND/protocol [19]; NCT04302766 treatment IND/protocol [20];

-Treatment protocol for healthcare professionals EAMS 11972/0002 remdesivir $100 \mathrm{mg}$ concentrate for solution for infusion [21]. -Treatment protocol for healthcare professionals_ EAMS 11972/0001 remdesivir $100 \mathrm{mg}$ powder for concentrate for solution for infusion [22]; -Treatment protocol for patients_EAMS 11972/0001 remdesivir $100 \mathrm{mg}$ powder for concentrate for solution for infusion [23];

-Treatment protocol for patients_EAMS 11972/0002 remdesivir $100 \mathrm{mg}$ concentrate for solution for infusion [24]; -Treatment protocol on the pharmacovigilance system -Information for medical directors [25]; -Early Access to Medicines Scientific Opinion-Public Assessment Report [26];

-Conditions of use, conditions for distribution and patients targeted, and conditions for safety monitoring addressed to member states for remdesivir available for compassionate use [16];

-Summary on compassionate use [27]; 
Table 2 Legal basis and regulatory evidence for emergency use of remdesivir in the USA

\begin{tabular}{lll}
\hline Emergency use of remdesivir & Legal basis & Regulatory evidence issued by FDA \\
\hline USA & Section 564 of the FD\&C Act amended: & • Fact Sheet for Health Care Providers [28]; \\
& -By the Project Bioshield Act of 2004; & • Fact sheet for patients and parent/caregivers Spanish [29]; \\
& -By the Pandemic and All-Hazards & • Frequently asked questions on the emergency use \\
& $\begin{array}{l}\text { Preparedness Reauthorization Act of 2013 } \\
\text { (PAHPRA), the 21st Century Cures Act of }\end{array}$ & authorization for remdesivir for certain hospitalized \\
& COVID, and Public Law 115-92 of 2017 & \\
&
\end{tabular}

remdesivir in COVID-19 pandemic was made possible through several regulatory approaches within the scope of clinical research and clinical practice [35, 37]. The primary suitable approach for accessing and investigating remdesivir were several clinical trials, on which patients within the eligibility criteria would be monitored properly and reliable and interpretable safety and efficacy data would be gathered. Meanwhile, patients who were not eligible to be enrolled in clinical trials would get access to remdesivir through compassionate use (expanded access, early access scheme) and emergency use $[16,26,28]$ as given in Table 3 . The approval of these regulatory approaches outside of clinical trials did not mean that remdesivir was safe and effective, and it was neither a formal approval nor a commitment for approval and not an authorization to provide remdesivir to patients.

The first individual compassionate use request for remdesivir was received on January 2020, to treat a 35year-old male COVID-19 patient in the USA [38], whereupon this approach was initiated and implemented by many other countries and in a larger number of patients [19]. The European Medicines Agency on 3 April 2020 provided not binding scientific recommendations and opinions for conditions of the use of remdesivir outside of clinical trials, requested from Greece, Estonia, Romania, and the Netherlands, recommending the target population, dosage, treatment duration, and a summary of the totality of the evidence [36]. Compassionate use of remdesivir in the EU was approved by national regulatory authorities of Switzerland, Spain, Slovenia, Hungary, Iceland, Greece, Italy, Netherlands, Portugal, Romania, Slovakia, Netherlands, Austria, Belgium, Germany, France, Estonia, and Cyprus [19]. This authorization was designated to treat a target group of COVID-19 patients not qualified for enrollment in clinical trials, for which European regulators would strictly review and track the safety data of remdesivir, rather than efficacy data [39]. Although most countries have similar regulatory requirements for enrollment to the compassionate use programs, there is no one-size-fits-all rule in terms of the legal basis, regulatory requirements, and terminology as given in Table 1. Compassionate use of Europe corresponds with expanded access in the USA designated and approved to be used for the treatment of individual patients, including in emergency use, for medium-size patent population and widespread use [40]. Hence, the compassionate use is not defined as a regulatory terminology by the Food and Drug Administration (FDA) [40]. Compassionate use of remdesivir in Europe is not designated for individual patients, because this approach falls within the remit of Article 5 of Directive 2001/83/EC also known as "Named Patient Basis," applicable in emergencies [41]. Also, two expanded access trials such as the Gilead's NCT04323761 [19] with 268 study locations to date, and the US army's NCT04302766 [20] of remdesivir do not have enough public data. This is especially in the context of study design and interventions to be further interpreted.

However, from Gilead's perspective, there are two terminologies and modalities such as "compassionate use" designated for individual patients and "expanded access" designated for a group of patients, stating that the manufacturer only accepts individual requests for pregnant women and children less than 18 years of age [35]. This is due to the process of transitioning in expanded access programs and the limited supply of the drug [35]. From the published original article of remdesivir's compassionate use on 10 April 2020, we have seen that there have been several issues not only in the methodology, study design, but also in the implementation of this program in clinics across USA, Canada, Japan, and the EU [31]. From 25 January to 7 March, the data of $53 \mathrm{pa}$ tients requiring invasive mechanical ventilation and ECMO were analyzed through this approach and clinical improvement was noted in 36 patients and a noncomprehensive safety report was available [31].

\section{The influence of results of clinical trials in expanding access to remdesivir}

Compared to other potential drugs for treating COVID19, remdesivir was accessed and investigated in fewer clinical trials. The multicenter double-blinded phase 3 RCT with a study design same as the National Institute of Allergy and Infectious Diseases (NIAID) trial (NCT04257656) reported inconclusive data due to the premature termination of COVID-19 outbreak in Hubei Province of China and the low-enrollment of patients [32]. Meanwhile, the open-label phase 3 RCT NCT04292899 published on 27 May 2020, did not show a significant difference between the 5-day course and 10-day course for COVID-19 patients not 

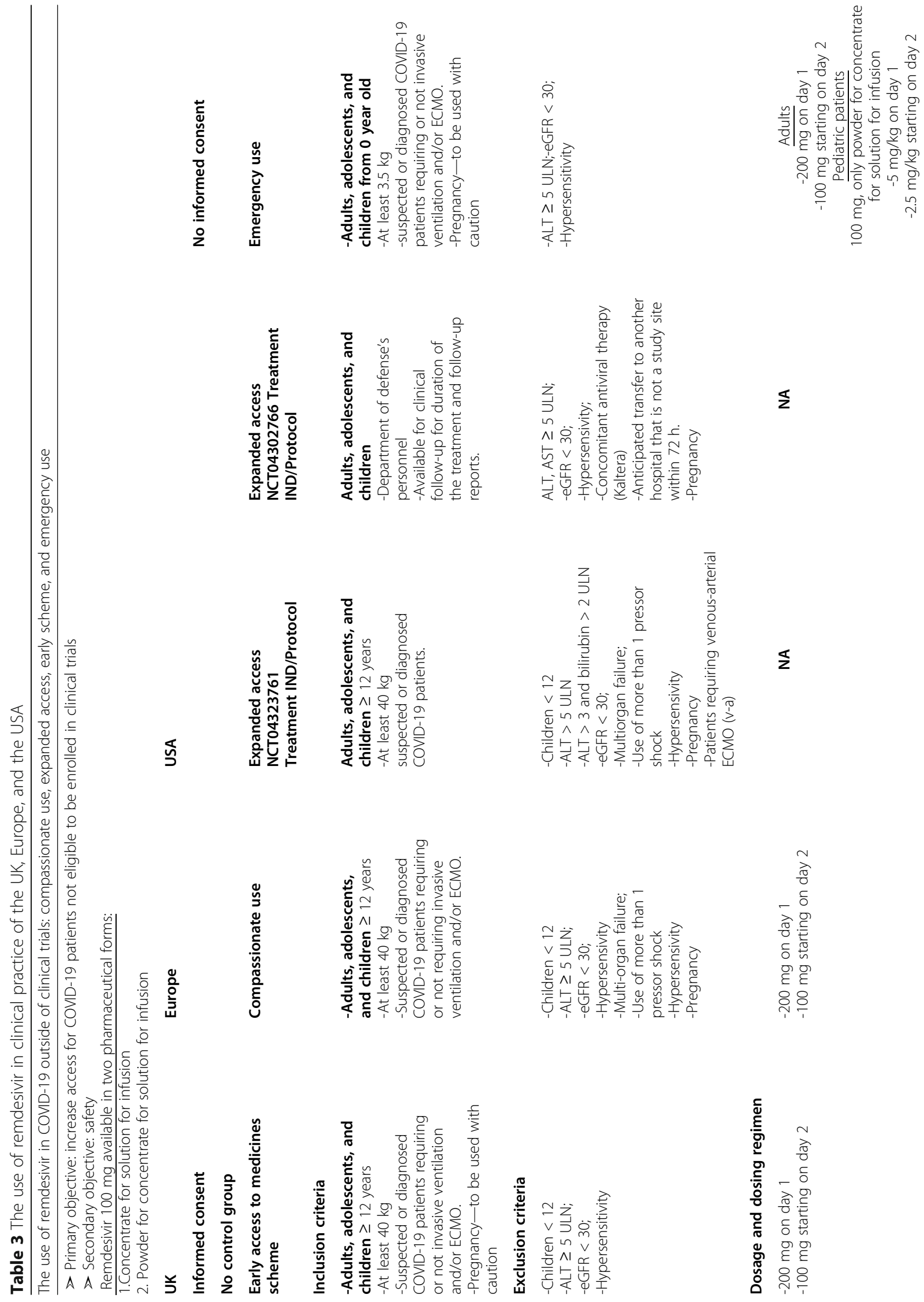


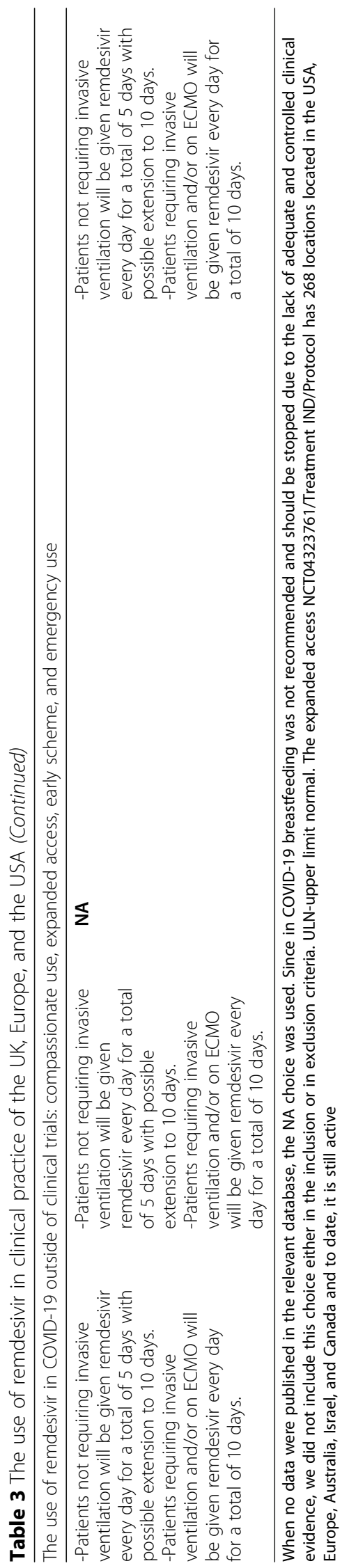


requiring invasive mechanical ventilation or ECMO in the baseline, while patients who would need invasive mechanical ventilation or ECMO, the treatment duration might be extended to 10 days [33]. Further interpretation of this trial would be of great significance, considering the limited medical supply and the high cost of remdesivir.

On the other hand, the National Institute of Allergy and Infectious Diseases (NIAID) double-blinded placebo trial NCT04280705, reported that remdesivir was superior to placebo in speeding up the recovery time in COVID-19 patients [34]. Remdesivir is enrolled also in the WHO solidarity clinical trial and INSERM discovery clinical trial. Nevertheless, there were still issues and gaps to be elucidated especially in terms of death rates, virus load data, and safety aspects [32-34]. However, from these preliminary data, the Food and Drug Administration (FDA) on 1 May 2020, approved its emergency use for patients at all ages requiring or not requiring invasive ventilation or ECMO [4]. Consequently, the European Medicines Agency (EMA) on 11 May 2020 revised the recommendations for compassionate use of remdesivir by extending the inclusion criteria with the addition of patients who do not require invasive ventilation to be treated for a period of 5 days through 10 days [16]. The Medicines and Healthcare products Regulatory Agency (MHRA) on 26 May 2020 approved early access scheme of remdesivir for patients of $\geq 12$ years requiring or not requiring invasive mechanical ventilation [17]. Since most of the RCTs excluded pregnant women and breastfeeding, the only therapeutic option for this group of patients was the use of remdesivir outside of clinical trials, although the EMA did not agree to use remdesivir in pregnant women at the beginning [16].

\section{Conditional approval and further implications}

In response to the current medical need for an effective therapeutic in context of public health crisis caused by COVID-19, the European Medicines Agency (EMA) on 30 April 2020 commenced a rolling review of the totality of evidence for remdesivir, as a potential therapeutic in the treatment of COVID-19 [42]. During this review, the EMA assessed the scientific dossier comprising of administrative, quality, preclinical, and clinical data proposed by Gilead and the available published literature and studies [43].

On 25 June 2020, the European Medicines Agency (EMA) issued a positive recommendation for the conditional approval of remdesivir in the European Union, although its safety and efficacy profile was not entirely elucidated [44]. Nevertheless, the EMA laid down explicit obligations in terms of quality, safety, and efficacy to be provided from Gilead, within a given deadline annexed at the European Public
Assessment Report of remdesivir [43]. The approval of remdesivir by the European Union on 3 July 2020 was mainly influenced by the outcomes from the pivotal NIAID trial [43]. Under this approval, remdesivir will be available only for pneumonia COVID-19 patients (12 years and older, weighing at least $40 \mathrm{~kg}$ ) requiring oxygen support in an initial dose of $200 \mathrm{mg}$ on day 1 followed by $100 \mathrm{mg}$ once daily on day $2-10$ [44]. Soon after the issuance of conditional approval of remdesivir in the $\mathrm{EU}$, the positive recommendation for early access scheme of remdesivir in the UK was withdrawn [45]. Mean while in the US, the emergency use authorization for remdesivir is still applicable [28]; Australia on 10 July 2020 issued a provisional approval for remdesivir after the recent approval from the EU, Japan, and Singapore [46]. To ensure equitable access for remdesivir in developing countries, the manufacturer Gilead is focused on advancing the manufacturing process and agreed to contract several generic developers to manufacture remdesivir [47]. To explore its potential to be used in the early stages of the disease and to avoid the hospitalization, the inhaled formulation of remdesivir is going to be evaluated in clinical trials [48]. Remdesivir is also under two clinical trials together with immunomodulators $[49,50]$ and under a clinical trial to evaluate the safety, tolerability, and pharmacokinetics in pediatric population [51]. Although on 10 July 2020, Gilead announced new additional information for remdesivir [52], still there is a need to accumulate more safety and efficacy data for the use of remdesivirespecially in children, pregnant women, breastfeeding, and patients with comorbidities.

\section{Conclusion}

COVID-19 pandemic has influenced large clinical trials to be conducted. As for the use of remdesivir outside of clinical trials, the attitudes of regulatory authorities across countries have been different from one to another, either in regulatory approvals or in protocols. Preliminary results of RCTs (mainly the NIAID trial) have altered the opinion of regulatory authorities on remdesivir, either by recommending or approving its use outside of clinical trials. Only after the clinical endpoints and other crucial characteristics of RCTs will be analyzed and interpreted, and further trials will generate more data about specific population, we will be able to see if regulatory authorities have acted well or otherwise. It is imperative to educate clinicians regarding regulatory mechanisms for early access of investigational drugs so that they understand the possibilities that their patients have in unmet medical situations. 


\section{Abbreviations}

EMA: European Medicines Agency; FDA: Food and Drug Administration; MHRA: Medicines and Healthcare Products Regulatory Agency; EBOV: Ebola virus; RCT: Randomized clinical trials; SARS-CoV: Severe acute respiratory syndrome coronavirus; MERS-CoV: Middle East respiratory syndrome coronavirus; ECMO: Extracorporeal membrane oxygenation; ECMO (vv): Veno-venous extracorporeal membrane oxygenation; ECMO (v-a): Venoarterial extracorporeal membrane oxygenation; ALT: Alanine aminotransferase; AST: Aspartate aminotransferase; eGFR: Estimated glomerular filtration rate; PAHPRA: the Pandemic and All-Hazards Preparedness Reauthorization Act; EAMS: Early access to medicines scheme; ULN: Upper limit normal; NIAID: National Institute of Allergy and Infectious Diseases

\section{Authors' contributions}

$\mathrm{VH}$ wrote and finalized the manuscript. AD, NR, IPS, MS,VP, AD, contributed with their clinical and scientific profile, AG gave guidance and approved this manuscript. The author(s) read and approved the final manuscript.

\section{Funding}

Not applicable

\section{Availability of data and materials}

Not applicable

\section{Competing interests}

Vesa Halimi, Armond Daci, Nevenka Ridova, Irina Panovska-Stavridis, Milena Stevanovic, Venko Filipce, Aleksandar Dimovski, and Aleksandra Grozdanova declare that no competing interests exist.

\section{Author details}

${ }^{1}$ Faculty of Pharmacy, University Ss. Cyril and Methodius University, Skopje, North Macedonia. ${ }^{2}$ Department of Pharmacy, Faculty of Medicine, University of Prishtina, Prishtina, Kosovo. ${ }^{3}$ University Clinic of Hematology, Medical Faculty, University Ss. Cyril and Methodius University, Skopje, North Macedonia. ${ }^{4}$ University Clinic of Infection Diseases and Febrile Conditions, Medical Faculty, University Ss. Cyril and Methodius, Skopje, North Macedonia. ${ }^{5}$ University Clinic for Neurosurgery, Medical Faculty, University Ss. Cyril and Methodius, Skopje, North Macedonia.

\section{Received: 3 July 2020 Accepted: 18 August 2020}

\section{Published online: 21 September 2020}

\section{References}

1. Halimi V, Daci A, Stojanovska S, Panovska-Stavridis I, Stevanovic M, Filipce V, et al. Current regulatory approaches for accessing potential COVID-19 therapies. J Pharm Policy Pract. 2020.

2. Food and Drug Administration (FDA). Letter revoking EUA for chloroquine phosphate and hydroxychloroquine sulfate. [Internet]. [cited 2020 July 19]. Available from: https://www.fda.gov/media/138945/download.

3. Recovery statement. Statement from the Chief Investigators of the Randomised Evaluation of COVid-19 thERapY (RECOVERY) Trial on lopinavirritonavir [Internet]. [cited 2020 July 19]. Available from: www.recoverytrial. net.

4. Food and Drug Administration (FDA). FDA COMBATING COVID-19 WITH THERAPEUTICS. [Internet]. [cited 2020 July 19]. Available from: https://www. fda.gov/media/136832/download.

5. Food and Drug Administration (FDA). Request for emergency use authorization for use of chloroquine phosphate or hydroxychloroquine sulfate supplied from the strategic national stockpile for treatment of 2019 coronavirus disease [Internet]. [cited 2020 July 19]. Available from: https:// www.fda.gov/media/136534/download.

6. Villar J, Ferrando C, Martínez D, Ambrós A, Muñoz T, Soler JA, et al. Dexamethasone treatment for the acute respiratory distress syndrome: a multicentre, randomised controlled trial. Lancet Respir Med. 2020.

7. Horby P, Lim WS, Emberson J, Mafham M, Bell J, Linsell L, et al. Effect of Dexamethasone in Hospitalized Patients with COVID-19: Preliminary Report. medRxiv. 2020.

8. Huet T, Beaussier H, Voisin O, Jouveshomme S, Dauriat G, Lazareth I, et al. Anakinra for severe forms of COVID-19: a cohort study. Lancet Rheumatol. 2020.
9. Cavalli G, De Luca G, Campochiaro C, Della-Torre E, Ripa M, Canetti D, et al. Interleukin-1 blockade with high-dose anakinra in patients with COVID-19, acute respiratory distress syndrome, and hyperinflammation: a retrospective cohort study. Lancet Rheumatol. 2020.

10. Liu T, Zhang J, Yang Y, Zhang L, Ma H, Li Z, et al. The Potential Role of IL-6 in Monitoring Coronavirus Disease 2019. SSRN Electron J. 2019:2020.

11. Xu X, Han M, Li T, Sun W, Wang D, Fu B, et al. Effective treatment of severe COVID-19 patients with tocilizumab. Proc Natl Acad Sci U S A. 2020.

12. Toniati P, Piva S, Cattalini M, Garrafa E, Regola F, Castelli F, et al. Tocilizumab for the treatment of severe COVID-19 pneumonia with hyperinflammatory syndrome and acute respiratory failure: A single center study of 100 patients in Brescia. Rev: Italy. Autoimmun; 2020.

13. Hung IFN, Lung KC, Tso EYK, Liu R, Chung TWH, Chu MY, et al. Triple combination of interferon beta-1b, lopinavir-ritonavir, and ribavirin in the treatment of patients admitted to hospital with COVID-19: an open-label, randomised, phase 2 trial. Lancet. 2020

14. Treon SP, Castillo JJ, Skarbnik AP, Soumerai JD, Ghobrial IM, Guerrera ML, et al. The BTK inhibitor ibrutinib may protect against pulmonary injury in COVID-19-infected patients. Blood. 2020.

15. Cao Y, Wei J, Zou L, Jiang T, Wang G, Chen L, et al. Ruxolitinib in treatment of severe coronavirus disease 2019 (COVID-19): A multicenter, single-blind, randomized controlled trial. J Allergy Clin Immunol. 2020.

16. European Medicines Agency (EMA). Annex I Conditions of use, conditions for distribution and patients targeted adressed to Member States Remdesivir Gilead. [Internet]. [cited 2020 Jul 19] Available online:https:// www.ema.europa.eu/en/documents/other/conditions-use-conditionsdistribution-patients-targeted-conditions-safety-monitoring-adressed_en-2. pdf.

17. Medicines and Healthcare products Regulatory Agency (MHRA). MHRA issues a scientific opinion for the first medicine to treat COVID-19 in the UK - GOV.UK [Internet]. [cited 2020 Jul 1]. Available from: https://www.gov.uk/ government/news/mhra-supports-the-use-of-remdesivir-as-the-firstmedicine-to-treat-covid-19-in-the-uk.

18. Agenzia Italiana del Farmaco (AIFA). Programmi di uso compassionevole COVID-19 | Agenzia Italiana del Farmaco [Internet]. [cited 2020 Jul 12] Available from: https://www.aifa.gov.it/programmi-di-uso-compassionevolecovid-19.

19. ClinicalTrials.gov National Library of Medicine (US).Identifier NCT04323761. Expanded Access Treatment Protocol: Remdesivir (RDV; GS-5734) for the Treatment of SARS-CoV2 (CoV) Infection (COVID-19) - Full Text View ClinicalTrials.gov [Internet]. [cited 2020 Jul 19]. Available from: https://www. clinicaltrials.gov/ct2/show/NCT04323761?term=remdesivir\&type= Expn\&cond=Covid-19\&draw $=2 \&$ rank $=1$.

20. ClinicalTrials.gov National Library of Medicine (US).Identifier NCT04302766. Expanded Access Remdesivir (RDV; GS-5734 ${ }^{\mathrm{TM}}$ ) - Full Text View ClinicalTrials.gov [Internet]. [cited 2020 Jul 19]. Available from: https://www. clinicaltrials.gov/ct2/show/NCT04302766.

21. Medicines and Healthcare products Regulatory Agency(MHRA). EAMS Information for HCP [Remdesivir 100 mg concentrate for solution for infusion] EAMS 11972/0002 Remdesivir 100 mg concentrate for solution for infusion Early Access to Medicines Scheme-Treatment protocol-Information for healthcare professionals. [Internet]. [cited 2020 Jul 1] Available from: https://assets.publishing.service.gov.uk/government/uploads/system/ uploads/attachment_data/file/887275/EAMS_11972_0002_TP_HCP_ Solution.pdf.

22. Medicines and Healthcare products Regulatory Agency (MHRA). EAMS Information for HCP [Remdesivir $100 \mathrm{mg}$ powder for concentrate for solution for infusion]. [Internet]. [cited 2020 Jul 1] Available from: https:// assets.publishing.service.gov.uk/government/uploads/system/uploads/ attachment_data/file/887276/EAMS_11972_0001_TP_HCP_Powder.pdf.

23. Medicines and Healthcare products Regulatory Agency (MHRA). EAMS Information for Patients [Remdesivir $100 \mathrm{mg}$ powder for concentrate for solution for infusion] . [Internet]. [cited 2020 Jul 1] Available from: https:// www.gov.uk/government/publications/early-access-to-medicines-schemeeams-scientific-opinion-remdesivir-in-the-treatment-of-patients-hospitalisedwith-suspected-or-laboratory-confirme/treatment-protocol-for-patients_ eams-119720001-remdesivir-100-mg-powder-for-concentrate-for-solutionfor-infusion.

24. Medicines and Healthcare products Regulatory Agency (MHRA). EAMS information for patients [remdesivir $100 \mathrm{mg}$ concentrate for solution for infusion] [Internet]. [cited 2020 Jul 1] Available from: https://assets. 
publishing.service.gov.uk/government/uploads/system/uploads/attachment_ data/file/887278/EAMS_TP_for_Patients_11972_0002_Solution.pdf.

25. Medicines and Healthcare products Regulatory Agency (MHRA). EAMS pharmacovigilance system [remdesivir $100 \mathrm{mg}$ powder for concentrate for solution for infusion and remdesivir $100 \mathrm{mg}$ concentrate for solution for infusion] EAMS 11972/0001 Remdesivir 100 mg powder for concentrate for solution for infusion EAMS 11972/0002 Remdesivir 100 mg concentrate for solution for infusion early access to medicines scheme-treatment protocolinformation on the pharmacovigilance system and requirements for reporting safety data [Internet]. [cited 2020 Jul 1] Available from: https:/assets. publishing.service.gov.uk/government/uploads/system/uploads/attachment_ data/file/887279/EAMS_TP_on_PVS_11972_0001_and_11972_0002.pdf:

26. Medicines and Healthcare products Regulatory Agency (MHRA). Early access to medicines scientific opinion-public assessment report product remdesivir $100 \mathrm{mg}$ powder for concentrate for solution for infusion remdesivir $100 \mathrm{mg}$ concentrate for solution for infusion [Internet]. [cited 2020 Jul 1]. Available from: https://assets.publishing.service.gov.uk/government/uploads/system/ uploads/attachment_data/file/897613/PAR_EAMS_11972_0001_AND_11 972_0002_PAR.pdf.

27. European Medicines Agency (EMA). Summary on compassionate use for Remdesivir Gilead [Internet]. [cited 2020 Apr 12]. Available from: https:// www.ema.europa.eu/en/documents/other/summary-compassionate-useremdesivir-gilead_en.pdf.

28. Food and Drug Administration (FDA). Fact sheet for health care providers emergency use authorisation (EUA) of remdesivir (GS-5734 ${ }^{T M}$ ) ). [Internet]. [cited 2020 Jul 4]. Available from: https://www.fda.gov/media/137566/ download.

29. Food and Drug Administration (FDA). Fact sheet for patients and parent/ caregivers emergency use authorization (EUA) of remdesivir for coronavirus disease 2019 [Internet]. [cited 2020 Jul 4]. Available from: https://www.fda. gov/media/137565/download.

30. Food and Drug Administration (FDA). Frequently asked questions on the emergency use authorization for remdesivir for certain hospitalized COVID19 patients. [Internet]. [cited 2020 Jul 4]. Available from:https://www.fda. gov/media/137574/download.

31. Grein J, Ohmagari N, Shin D, Diaz G, Asperges E, Castagna A, et al. Compassionate Use of Remdesivir for Patients with Severe Covid-19. N Engl J Med. 2020.

32. Wang $Y$, Zhang D, Du G, Du R, Zhao J, Jin Y, et al. Remdesivir in adults with severe COVID-19: a randomised, double-blind, placebo-controlled, multicentre trial. Lancet. 2020.

33. Goldman JD, Lye DCB, Hui DS, Marks KM, Bruno R, Montejano R, et al. Remdesivir for 5 or 10 Days in Patients with Severe Covid-19. N Engl J Med [Internet]. Massachusetts Medical Society; 2020 [cited 2020 Jun 5]; NEJMoa2015301. Available from: http://www.nejm.org/doi/10.1056/NEJMoa2 015301.

34. Beigel JH, Tomashek KM, Dodd LE, Mehta AK, Zingman BS, Kalil AC, et al. Remdesivir for the treatment of Covid-19 — preliminary report. N Engl J Med [Internet]. Massachusetts Medical Society; 2020 [cited 2020 Jun 5]; NEJMoa2007764. Available from: http://www.nejm.org/doi/10.1056/NEJMoa2 007764.

35. Gilead Sciences. Gilead Sciences Statement on Access to Remdesivir Outside of Clinical Trials [Internet]. [cited 2020 Jun 2]. Available from: https://www.gilead.com/news-and-press/company-statements/gileadsciences-statement-on-access-to-remdesivir-outside-of-clinical-trials.

36. European Medicines Agency (EMA). EMA provides recommendations on compassionate use of remdesivir for COVID-19 | European Medicines Agency [Internet]. [cited 2020 Jun 4]. Available from: https://www.ema. europa.eu/en/news/ema-provides-recommendations-compassionate-useremdesivir-covid-19.

37. European Medicines Agency. Treatments and vaccines for COVID-19 | European Medicines Agency [Internet]. [cited 2020 Apr 21]. Available from: https://www.ema.europa.eu/en/human-regulatory/overview/public-healththreats/coronavirus-disease-covid-19/treatments-vaccines-covid-19.

38. Holshue ML, DeBolt C, Lindquist S, Lofy KH, Wiesman J, Bruce H, et al. First case of 2019 novel coronavirus in the United States. N Engl J Med. 2020.

39. European Union; European Medicines Agency. Guideline on compassionate use of medicinal products, pursuant to Article 83 of Regulation (EC) No 726/ 2004. 2007; Available from: https://www.ema.europa.eu/en/documents/ regulatory-procedural-guideline/guideline-compassionate-use-medicinalproducts-pursuant-article-83-regulation-ec-no-726/2004_en.pdf;.
40. Food and Drug Administration. Expanded access to investigational drugs for treatment use - questions and answers [Internet]. 2017. p. 1473-4. Available from: https://www.fda.gov/media/85675/download;:

41. European Parliament and the Council of the European Union. Directive 2001/83/EC of the European Parliament and of the Council of 6 November 2001 on the Community code relating to medicinal products for human use [Internet]. Off. J. L 311 , 28/11/2001 P. 0067 - 0128; OPOCE; [cited 2020 Jun 9]. Available from: https://eur-lex.europa.eu/legal-content/EN/TXT/ HTML/?uri=CELEX:32001L0083\&from=EN.

42. European Medicines Agency (EMA). EMA starts rolling review of remdesivir for COVID-19 | European Medicines Agency [Internet]. [cited 2020 Jun 12]. Available from: https://www.ema.europa.eu/en/news/ema-starts-rollingreview-remdesivir-covid-19.

43. European Medicines Agency (EMA). European Public Assessment Report (EPAR) of Venklury (remdesivir) [Internet]. [cited 2020 Jun 9].Available from: www.ema.europa.eu/contact;

44. European Medicines Agency (EMA). First COVID-19 treatment recommended for EU authorisation | European Medicines Agency [Internet]. [cited 2020 Jul 19]. Available from: https://www.ema.europa.eu/en/news/ first-covid-19-treatment-recommended-eu-authorisation.

45. Medicines and Healthcare products Regulatory Agency (MHRA). [Withdrawn] Early access to medicines scheme (EAMS) scientific opinion: remdesivir in the treatment of patients hospitalised with suspected or laboratory-confirmed SARS-CoV-2 infection who meet the clinical criteria - GOV.UK [Internet]. [cited 2020 Jul 19]. Available from: https://www.gov.uk/government/publications/ early-access-to-medicines-scheme-eams-scientific-opinion-remdesivir-in-thetreatment-of-patients-hospitalised-with-suspected-or-laboratory-confirme;

46. The Therapeutic Goods Administration (TGA). Australia's first COVID treatment approved | Therapeutic Goods Administration (TGA) [Internet]. [cited 2020 Jul 19]. Available from: https://www.tga.gov.au/media-release/ australias-first-covid-treatment-approved.

47. Gilead Sciences. An Open Letter from Daniel O'Day, Chairman \& CEO, Gilead Sciences [Internet]. [cited 2020 Jul 19]. Available from: https://stories.gilead. com/articles/an-open-letter-from-daniel-oday-june-29.

48. Gilead Sciences. An Open Letter from Daniel O'Day, Chairman \& CEO, Gilead Sciences [Internet]. [cited 2020 Jul 19]. Available from: https://stories.gilead. com/articles/an-open-letter-from-daniel-oday-june-22.

49. U.S. National Library of Medicine Clinicaltrials.gov. A study to evaluate the efficacy and safety of remdesivir plus tocilizumab compared with remdesivir plus placebo in hospitalized participants with severe covid-19 pneumonia - full text view - ClinicalTrials.gov [Internet]. [cited 2020 Jul 19]. Available from: https://www. clinicaltrials.gov/ct2/show/NCT04409262?term=remdesivir\&draw=3\&rank=11.

50. U.S. National Library of Medicine ClinicalTrials.gov. Adaptive COVID-19 Treatment Trial 2 (ACTT-2) - Full Text View - ClinicalTrials.gov [Internet]. [cited 2020 Jul 19]. Available from: https://www.clinicaltrials.gov/ct2/show/ NCT04401579?term $=$ remdesivir\&draw $=2 \&$ rank $=13$.

51. U.S. National Library of Medicine ClinicalTrials.gov. Study to evaluate the safety, tolerability, pharmacokinetics, and efficacy of remdesivir (GS-5734 ${ }^{\mathrm{TM}}$ ) in participants from birth to $<18$ years of age with coronavirus disease 2019 (COVID-19) - full text view - ClinicalTrials.gov [Internet]. [cited 2020 Jul 23]. Available from: https://clinicaltrials.gov/ct2/show/NCT04431453.

52. Gilead Sciences. Gilead presents additional data on investigational antiviral remdesivir for the treatment of COVID-19 [Internet]. [cited 2020 Jul 19]. Available from: https://www.gilead.com/news-and-press/press-room/pressreleases/2020/7/gilead-presents-additional-data-on-investigational-antiviralremdesivir-for-the-treatment-of-covid-19.

\section{Publisher's Note}

Springer Nature remains neutral with regard to jurisdictional claims in published maps and institutional affiliations. 